\title{
Research of the Intelligent Control Development Trends of Farm Grasping Grass Machine
}

\author{
Zhen-hang Cao, Guan-feng Li, Zhong-xu Dou \\ College of Mechanical and Electrical Engineering, Henan Agricultural University, Zhengzhou, \\ Jinshui district, China \\ College of Mechanical and Electrical Engineering, Henan Agricultural University, Zhengzhou, \\ Jinshui district, China \\ College of Mechanical and Electrical Engineering, Henan Agricultural University, Zhengzhou, \\ Jinshui district, China \\ 13526728677@163.com, li-guanfeng@163.com,553156572@qq.com
}

\begin{abstract}
Keywords: Grasping Grass Machine; Intelligent Control; Development Trend.
Abstract. In this paper the problems is analyzed, that the phenomenon of burning crops straw, although it is prohibited times without number, and which causing serious air pollution are a great deal of occurrences in recent years in rural area. It points out that one of the main reasons is lacking efficient machine for collecting and loading straw, so that the comprehensive utilization of straw is stopped at source. In order to reduce air pollution and improve energy utilization, by looking at data and on-the-spot investigation. Based on this, the current situation of farm grasping grass machine and the related demand of intelligent control technology are studied, and the development tendency is discussed. For the development needs of intelligent control of farm grasping grass machine, five major directions are suggested, bus-oriented, polarized, seriation, networked, greening.
\end{abstract}

\section{Introduction}

China is a large agricultural country, crop straw resources are very abundant that the total amount of produced annually by 600 to 800 million tons. However, these straw as a valuable resources and there are still many problems in the use of process, leading to large straw are directly burned or discarded. Analyzing the main reason, the more difficult problems is how to collect the straw easily. The traditional collecting way is mostly rely on manpower, which spend a large number of time and labor, not only labor-intensive, low efficiency, high labor intensity, high cost, but also concentrate transport and storage are difficult. With the accelerating process of urbanization is expediting, a large number of rural laborers move to cities, the phenomenon of aging of the agricultural workers and feminization is getting worse, there is not enough labor to collect and disposal, so that the primitive and simple manner-incinerated were forced to adopt. The mode of burning straw not only are the civil aviation, transportation and other departments affected, but also the environment has been suffered seriously pollution. In recent years, although the country has a strict management and all levels of government has take a variety of measures to prohibit burning straw, there is always forbidden and hardly stops, the contamination events of caused by the burning of straw have occurred. Among which of the key is lack of a suitable mechanical equipment for loading and collecting crop straw, thus the depth use of straw is being impeded. Therefore, farm grasping grass machine has been pay attention to and developed in recent years. The development and popularization and application of grasping grass machine have effectively solved which our crops are harvested after the issue of straw collecting and utilizing to provide a powerful support. However, with the gradual expansion of market demand and enhance the comprehensive performance of its work requirements, the traditional and simple of machinery operation function would be not satisfied developing requirements, improving the intelligent control of farm grasping grass machine would have been become a trend. Therefore, this paper will discuss the intelligent development trends of the machine.

\section{The requirement of intelligent control for farm grasping grass machine}

Researching the technology of intelligent engineering machinery can be traced back to the beginning of 1990 s in china, the country have launched the "863 plan" and conducted the research of technology of engineering machinery robot that the initiative of in the field of automation of scientists Jiang 
Xinsong[1]. In recent years, the research of intelligent control has also become active and emergence of the boom, many universities and research institutes are actively developing research and it has achieved a number of important achievements besides convening some of related to the academic conferences of intelligent control, intelligent control as an independent emerging discipline which is being formed in the country [2]. The intelligent control technology of emerging has also been widely applied to all aspects of people's lives, which are also constantly deepening in various types of machinery of application. However, intelligent control is only just beginning for farm grasping grass machine as nascent machinery.

Farm grasping grass machine is a loading machinery to which is specially used grab grass fodder or crop straw, and other rod-shaped objects, it is a equipment to which is mainly used the collect transport of wheat, corn, sorghum, soybeans and other crops straw that can also as the biomass energy power plants, paper mills, and straw acquisition of plants of stacking, handling, loading, clearance. Currently, the research and development of farm grasping grass machine is still infancy, it has mostly remained the design and innovation of in the form of gripper head fetch and grasp arm drive, and intelligent control is blank that is far less than the extent of intelligent.

Grasping grass machine as a kind of special loader which the existing of loader application of control technology can also be transplanted. Currently, the intelligent control technology of grasping grass machine to which can be mainly used the following areas:

\section{Power system}

The power system includes electronic control system of power, electric control system of diesel engine, control system of fuel and random automatic protection system.

The electronic control system of power is based on the status of the work of gearing and hydraulic system that is automatically adjust to the engine output in order to meet the needs of different operating conditions and improve the economy of fuel.

Diesel engine electronic control system equipment is used to fuel injection for control and maintain the engine electronic control modules of optimum performance that can also communicate with the detection system when a malfunction occurs to alert drivers attention.

The main role of the fuel control system is reasonably to control fuel injected and intake air amount so that the fuel and air of mixture ratio reach the best and improve the efficiency of fuel and ensure the engine discharge exhausts comply with the regulation control of environment.

Random automatic protection system includes an engine automatic control, antiskid control system. Among of them, the role of the engine automatic control system is automatically drop the speed of engine and reduce the consumption of fuel and engine noise when the loader is a non-working condition.

\section{Transmission system}

Transmission systems mainly have load sensing transmission system and steering transmission of integration control system.

Load sensing transmission system is automatically adjust to the speed and engine flywheel of torque according to the status of load in order to achieve the power output of high-speed, low torque or low-speed, high torque.

Steering transmission of integration control system is eliminate of the traditional steering wheel and shift lever, the steering wheel and transmission operating device are integrated into one operating handle which is adopted simple of trigger directional control switch and gear selector of separate loading accelerate button. Using the natural of toggle movement is flip the operating lever left and right to achieve steering; the use of thumb selection is achieve forward and backward, acceleration and deceleration. 


\section{Running system}

Running systems have drive stability control system, adhesion control system, comfort-driven control system, automatic transport control and load of automatic stabilizers, etc.

Drive stability control system there is an accumulator increase in the movable arm lift hydraulic cylinder and circuit in order to attenuate the vibration of produced by the process of working device in the machine of moving and reduce the bumps of loader.

Adhesion control system is install a speed sensor on each wheels that is automatically force to required braking for wheels and the torque is conveyed to the closed of wheels so that loaders is straight running and turning.

Comfort-driven control system is aims to improving the driver's comfort and helping the long periods of time working drivers reduce labor and keep operating efficiency.

Automatic transport control system can reduce the vibration of machine on uneven roads and improve driving comfort and operational efficiency.

Load of automatic stabilizers is use a pair of steel die nitrogen accumulators communicate with the working device hydraulic system that is mounted the front frame. The system is automatically disconnect when the job or at low speed; the solenoid valve of electronic speed control switch is automatically open and the accumulators is absorb the vibration and shock loads of working device hydraulic system and improve the stability of operation, safety and comfort when the speed exceeds a set value[3].

\section{The display system with intelligent instrument}

Display system with intelligent instruments included: key information display system, Panel control system and computer monitoring and control system, and so on.

Panel control system: It is adopting control panel and it contained the sound, light, electric and digital display, it achieved alarm and monitoring by loading equipment and instrument.

Key information display system: It can be real-time monitoring and control the loader's job status by the network communication technology in the office, thus to provide the driver accurate fault diagnosis information based on text tip.

Computer monitoring and control system: It can be continuous monitoring more than ten performance parameters of the loader. In case of emergency, it can remind the driver of it by the way of acoustic generator's auditory signal and the liquid crystal display. Achieve the combination of auditory and visual effect, to reduce the potential safety hazard and the accident rate.

\section{Remote control system}

Remote control system included remote control operating system and satellite control system.

Remote control operating system: It operating the loader by the remote control for realizing unmanned vehicles. This technology can be applied to some dangerous environments, such as high temperature place, rescue work, nuclear waste cleanup and underground mining. Not only does it reduce the risk of the worker, but it can make many significant social benefits increase. For example, the Sierrita Cat992C type wheel loader had installed the remote monitoring system which was developed by the Black Box Automation Company [3].

Satellite control system: It mainly includes GPS satellite positioning system and satellite communication system. GPS satellite positioning system can determine the current location of the loading machine; Satellite communication system can be monitoring and managing the loading machine. 


\section{Other intelligent system}

Not only can grasping grass machine use the above several aspects of the control technology, but it also can apply to these control technology:

Fault diagnosis system: It reminds the driver of some potential problems through the indicator lights on the control panel and the alarm signal which includes auditory and visual. The driver just was so absorbed in his work without need to often check instrument parameters.

Electronic control of equipment: It includes the electronic control system of the movable arm automatically lifting limit, the electronic control system of the mechanical paw automatically come back, the electronic control system for reducing driving vibration and Payload measuring system, etc.

Pin shaft lubrication system: It can provide lubrication service for pin shaft within work device, and make the pin shaft's assignments easy to complete.

Snatch weighing system: It can automate weighing and display the net load of the goods, so that the driver can prevent machine overloading and underloading. The number of invalid sports would be decreased by driver. In this way, it can save operation time and improve production efficiency.

Hydraulic pump electronic management system: It can automatically monitor the running state of hydraulic pump [4].

The control technologies of the above had been widely used in loaders at home and abroad, and transplanted these technologies in the grasping grass machine is completely feasible.

\section{The development trend of the farm grasping grass machine}

\section{The field bus}

At present, the field bus has become the important manifestation of the development of automation. The application of field bus technology, can organize the centralized and distributed test system to easier. With the development of the grasping grass machine, and its intelligent control system should become more perfect, concentration control will be increasingly unable to meet the complex, the remote and wide range of measurement and control task. And then, it must form a field instrument network for data sharing that is the field bus control system (FCS). It is a kind of open, full digital, bidirectional and multi-site communication system which has been used between field intelligent instruments and the central control, and it can make the measurement and control instrument to achieve further high precision, high stability, high reliability, high adaptability and low consumption [5].

CAN (Controller Area Network) is a kind of field bus that is high performance, high reliability, easy development and low cost. CAN bus is a kind of serial data communication bus, so that the data exchange problem of intelligent control system can be solved between all parts of the measurement control units. The control system of the grasping grass machine will be designed On the basis of the CAN bus.

\section{Polarization}

The future of grasping grass machine will be developing in the direction of polarization, and it mainly contains these two main directions: big and small.

"Big" means that grasping grass machine will develop to high power and large-scale. Along with the advancement of rural land contracting mode, scale management has become a trend, and operation will need to match the large-scale agricultural machinery equipment. Accordingly, the grasping grass machine is also toward to large-scale development, so as to adapt to the future development direction of the new countryside.

"Small" mainly means that we should produce portable flexible and economical tiny grasping grass machines.Land resources are rich in our country, and the topography is varied, not a place does the 
heavy machinery arrived, small machinery can go. It is light, flexible, economical, practical and easy to start so that some current users more like it [6].

\section{Form series of products}

At present, electronic products have been formed series. In daily life, we often use mobile phones, televisions, refrigerators, cars, etc, and they have formed series products. The trend of the development of the grasping grass machine is also to the series.

The advantages of series Products: On the one hand, the grasping grass machine can be produced in a large scale, and it could improve manufacturing efficiency, so as to reduce the production cost; On the other hand, the grasping grass machine can form a complete industry chain which contains production, sales and after-sales service, and after-sales maintenance is c convenient. It can improve reputation of the enterprise, expand the influence and enhance competitiveness.

\section{The technical parameters of network}

Technical parameter of the network is that synthetically utilize the network carrier to build a grasping grass machine public network communication platform that is shared medium base on the Internet. Companies can make their own series of protects' technical parameters of the grasping grass machine sharing on the network platform, Take the advantage of others, make up for their own shortcomings. This way can help to accelerate the development of the grasping grass machine, promote it rapid upgrade.

With the development of the information network technology, network-based technologies constantly updated, more and more software to make our life more convenient, thus to the grasping grass machine to rapid development cannot leave the network's support.

\section{The road of green industry}

The green industrial road of the farm grasping grass machine is embodied in two aspects: one is the manufacturing enterprises of the grasping grass machine to achieve the national standard, realize clean production; second is that indexes of the grasping grass machine to meet the national requirements, to reduce the pollution of the atmospheric environment.

At present, although the green manufacturing is still primary in our country, the green manufacturing of the grasping grass machine should keep up with the development of the times. Enterprises should put the development of green manufacturing technology to be one of the main tasks and responsibilities as technology promotion, to the actual needs of production and technology development as the guidance, adopt corresponding way of green manufacturing for production, and take the path of the green industry, to achieve sustainable development, to realize the economic and social benefits synchronous growth.

Due to the industrial production requirement for the quality of the finished product is increasing day by day, the national policy and law of energy conservation and emissions reduction have specific requirements and regulations. Therefore, we improve the grasping grass machine's emissions standard that is the inevitable requirement, which requires the overall fabrication process of the grasping grass machine to keep up with the needs of the development. Not only we can save energy, but achieve non-pollution emission.

\section{Conclusions}

With the progress of science and technology, intelligent control technologies have penetrated into all fields which have included the modern industry, agriculture and national defense, etc. As a result, agricultural machinery will develop to the trend of intelligent that is inevitable. With the increase rate of new rural construction and national attention to the environmental protection, the agricultural 
renewable resources utilization will further enhance. The grasping grass machine as key equipment of comprehensive utilization of crop straw, it is the special machine that serviced agricultural production for the full mechanization, bears its own unique tasks and mission. The intelligent control technology can improve its comprehensive performance. There is no doubt that the intelligent control technology will be to the main research content of the future development of the grasping grass machine.

This paper Analyzed intelligent control technologies which can be used for the farm grasping grass machine, I hope these tips are certain guidance and reference value to the future farm grasping grass machine's technology improvement and development.

\section{Reference}

[1] Guo-xiang $\mathrm{Wu}, \mathrm{Yu}-\mathrm{he} \mathrm{Li}$. Intelligent engineering machinery and the informationization development situation [J]. Journal of Construction Machinery, 2013, 44 (1) : 2-7.

[2] Shun-huang Wang, Di-qian Shu. Intelligent control system and application [M]. Beijing: Mechanical Industry Press, 2005:1-10, 10-20.

[3] Zhan-min Yang, Zhi-ming Wang, Chun-qiu Zhang, etc. Wheel loader [M]. Beijing: Chemical Industry Press, 2006:1-9272-305.

[4] Pei-yin Luo, Kai Liao. Analyses the buildup of an intelligent control system of loader [J]. Journal of Construction Machinery, 2007, (38) : 4 to 6.

[5] Shan-feng Shao, Guo-xiang Wu, Yu-he Li, Ying-ying Li. Research of the intelligent control system of loader [J] Journal of Construction Machinery, 2009, 40 (3) : 4 to 6.

[6] Yi Huang. The future trend of agricultural development will be polarised [J]. Friends of The Agricultural Machinery, 2004, 2 (7) : 52. 